\title{
Restrukturierungsprozesse einer Stadt-Umland-Ökonomie: Veränderung der Wirtschaftsstruktur im Agglomerationsraum Basel
}

\author{
Andrea Kampschulte, Renato Strassmann, Basel \\ 1 Globale und regionale \\ Restrukturierungsprozesse
}

Globale, ökonomisch-soziale Restrukturierungsprozesse bestimmen seit den 70er Jahren die Entwicklung von Regionalstrukturen, Städtesystemen und Stadt-Umland-Beziehungen sowie innerstädtischen Wirtschafts- und Sozialgefügen. $\mathrm{Zu}$ den Merkmalen des «neuen makroökonomischen Entwicklungsmusters» zählen nach KRÄTKE (1996: 16 ff.):

\section{- Flexibilisierung und Internationalisierung} von Produktion und Kapitalverwertung

Verschärfter Standortwettbewerb, verlangsamtes Wachstum, zunehmend instabile Märkte sowie fortschreitende Differenzierung und Aufspaltung von Konsummustern erfordern flexiblere Produktionsweisen und die «Verschlankung» von Unternehmen. Die fortschreitende Internationalisierung und Kapitalkonzentration im Unternehmenssektor ermöglichen eine stärkere räumliche Aufgliederung von Betriebsteilen und Unternehmensfunktionen. Es kommt zu einer «Flexibilisierung der Standortwahl». Folgen sind der Bedeutungszuwachs der Städte als Direktions- und Kontrollzentren sowie als Standort unternehmensorientierter Dienstleistungen (SASSEN 1996: 20).

- Flexibilisierung und Aufspaltung der Lohn- und Beschäftigungsverhältnisse

Flexible Produktionsmodelle und instabile Absatzmärkte erfordern eine steigende Anpassungsfähigkeit der Arbeitnehmer an zeitlich befristete Arbeit, Teilzeitarbeit und flexible Arbeitszeiten (SASSEN 1996: 139). Beim Zweistufensystem nach amerikanischem Vorbild wird eine Stammbelegschaft von Dauer- und Vollzeitbeschäftigten durch Teilzeit- und Zeitbeschäftigte verstärkt (RIFKIN 1996: 134). Folgen der deregulierten flexiblen Beschäftigungsverhältnisse sowie der allgemeinen Entkopplung von Produktivitäts- und Einkommensentwicklung sind wachsende Einkommensunterschiede und Massenarbeitslosigkeit.

- Neue Regulationsweisen mit verstärkter Marktsteuerung, Flexibilisierung und Deregulierung Die Deregulierung überkommener politischinstitutioneller Steuerungsmechanismen und der
Rückbau des Sozialstaates führen im Produktionsbereich zu neuartigen Verflechtungs- und Organisationsbeziehungen. Es entstehen flexible Netzwerke kleiner, unternehmerisch autonomer Teams (LuTz 1997: 130).

\section{Grundlagen der Untersuchung}

\subsection{Ziel und Arbeitshypothesen}

Eine empirische Überprüfung dieser theoretisch und eher im makroökonomischen Bereich konzipierten Thesen steht für den lokalen/regionalen Bereich weitgehend noch aus. Dieser Beitrag untersucht daher anhand ausgewählter Indikatoren, inwieweit die genannten Restrukturierungsprozesse auch in Basel zu beobachten sind und zur Veränderung des Wirtschaftsraumes beitragen. Im Mittelpunkt stehen die Flexibilisierung der Produktion und des Arbeitsmarktes. Aus den skizzierten makroökonomischen Entwicklungstendenzen werden folgende Arbeitshypothesen abgeleitet:

- Flexible/«schlanke» Produktion setzt weniger Ressourcen ein als Massenfertigung. Dies führt zu einem allgemeinen Rückgang der Beschäftigtenzahlen und einer Zunahme der Arbeitslosigkeit.

- Die Beschäftigung verlagert sich vom sekundären zum tertiären Sektor.

- Im tertiären Sektor kommt es zu einer Umschichtung zugunsten der höherwertigen, unternehmensorientierten Dienstleistungen.

- Die Flexibilisierung der Beschäftigungsverhältnisse führt $\mathrm{zu}$ einer Zunahme der Teilzeitbeschäftigten.

- Bezüglich der Betriebsgrössen führt die Flexibilisierung zu einer Verschiebung zugunsten der Klein- und Mittelbetriebe.

- Räumliche Dekonzentrationsprozesse vom Zentrum in das Umland zeichnen sich im Produktionsbereich (z. B. chemische Industrie, Maschinenbau, Einzelhandel) ab.

- Räumliche Konzentrationsprozesse sind für die höherwertigen Dienstleistungen wie Banken oder unternehmensbezogene Dienstleistungen zu beobachten.

\subsection{Abgrenzung des Untersuchungsgebietes}

Mit Umland wird der Raum um eine Stadt bezeichnet, der intensive sozioökonomische Verflechtungen mit dem Zentrum aufweist und/oder in 
den Prozess der Suburbanisierung einbezogen ist. Der Definition des Stadt-Umland-Raumes gemäss entspricht dieser weitgehend einem Agglomerationsraum (LESER [Hrsg.] 1997: 920,17). Zur Festlegung des Untersuchungsgebietes wird daher auf eine Abgrenzung der Agglomeration Basel zurückgegriffen, die zahlreiche sozioökonomische Kriterien, wie Bevölkerungsdichte, Wanderungssaldo, Altersstruktur, Ausländeranteil, Bildungsstruktur, Haushaltsstruktur und Steuererträge pro Kopf, einbezog (SchneIder-SLIWA, KampschUlte, Nommel, Sandtner, Strassmann \& WaffenSCHMIDT 1999). Zum Umland der Kernstadt Basel zählen danach die Gemeinden Riehen und Bettingen (Kanton Basel-Stadt) sowie die zum Kanton Basel-Landschaft gehörigen Gemeinden Allschwil, Binningen, Bottmingen, Reinach, Münchenstein, Muttenz, Birsfelden und Pratteln (innerer Agglomerationsgürtel, Karte 1) sowie Schönenbuch, Oberwil, Biel-Benken, Therwil, Ettingen, Aesch, Arlesheim, Frenkendorf, Füllinsdorf und Augst (äusserer Agglomerationsgürtel). Alle übrigen städtischen und ländlichen Gemeinden des
Kantons Basel-Landschaft gehören zum ländlichen Raum und damit nicht mehr zum Untersuchungsgebiet.

Der Untersuchungsraum umfasst eine Fläche von 14989 ha. Bei einer Zahl von 362780 Einwohnern (1995) ergibt sich eine Bevölkerungsdichte von 24,2 Personen/ha. Sie nimmt von der Kernstadt mit dem höchsten Dichtewert (73,6 Einwohnern/ha) zum Agglomerationsrand hin kontinuierlich ab. Die Hauptachsen der Siedlungs- und Verkehrsentwicklung sind die sternförmig nach aussen verlaufenden Täler von Birs, Birsig, Rhein und Ergolz, die auch die Hauptgliederungselemente der Wirtschaftslandschaft darstellen.

\section{Wirtschaftliche Strukturmerkmale der Stadt-Umland-Ökonomie Basel 1995}

\subsection{Beschäftigungsstruktur}

Die Anzahl der Voll- und Teilzeitbeschäftigten im Untersuchungsgebiet betrug 233554 Personen (1995), wovon 75314 Personen im sekundären

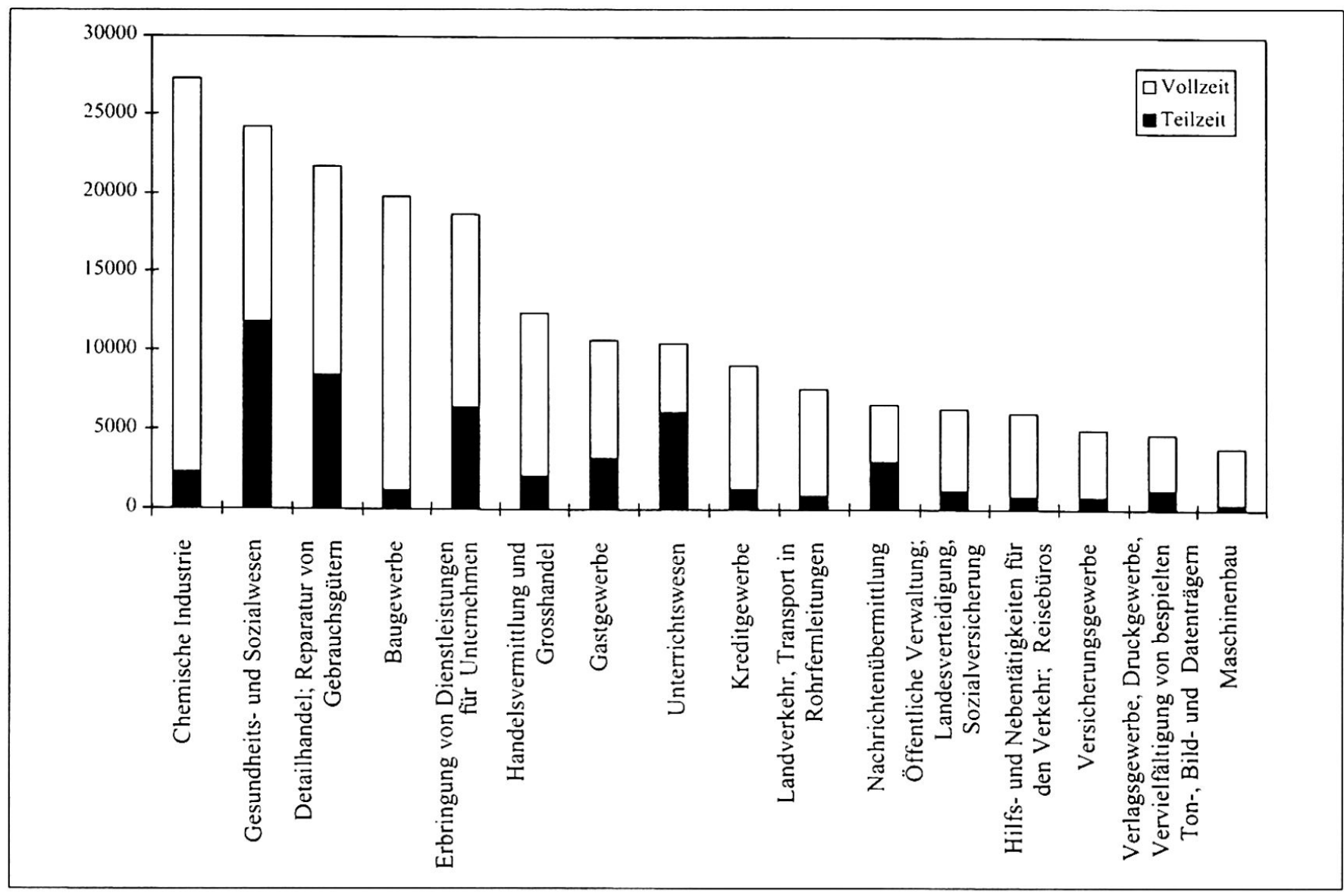

Abb. 1: Voll- und Teilzeitbeschäftigte in den wichtigsten Branchen des Untersuchungsgebietes 1995 Full-time and part-time employment in the most important branches of the study area in 1995

Emplois à plein-temps et emplois à temps partiel dans les branches les plus importantes au territoire de l'enquête 1995

Quelle: Statistisches Amt Des Kantons Basel-Landschaft [Hrsg.] (1997): Betriebszählung 1995. 
Sektor und 158240 Beschäftigte im tertiären Sektor tätig waren. Mit einem Anteil von $67,8 \%$ an der Gesamtbeschäftigung (Sektor II und Sektor III) lag der Beschäftigtenschwerpunkt somit im tertiären Sektor.

Die räumliche Verteilung der Beschäftigten zeigt Konzentrationen entlang eines parallel zum Rhein verlaufenden Bogens, der von Basel über Münchenstein, Muttenz und Pratteln nach Augst reicht. Die höchste Beschäftigungsdichte erreicht die Kernstadt mit 880 Beschäftigten pro 1000 Einwohner. Minima weisen die Gemeinden Ettingen und Riehen mit 200 Beschäftigten pro 1000 Einwohner auf. Zu den überwiegend industriell geprägten Gemeinden (Beschäftigtenanteile um 50\% ) zählen Aesch, BielBenken, Ettingen, Muttenz, Augst und Pratteln. Beschäftigungsschwerpunkte im tertiären Sektor (Beschäftigtenanteile um 70\%) weisen die Stadt Basel sowie die Gemeinden Riehen, Arlesheim, Binningen, Bottmingen, Oberwil und Schönenbuch auf.

Im Branchenspektrum dominieren die Chemie $(11,7 \%)$, das Gesundheits- und Sozialwesen $(10,4 \%)$, der Detailhandel und die Reparatur von Gebrauchsgütern (9,3\%), das Baugewerbe (8,5\%), die Erbringung von Dienstleistungen für Unternehmen $(8,0 \%)$ sowie die Handelsvermittlung und der Grosshandel mit einem Anteil von 5,3\% an den Beschäftigten (Abb. 1). Die sechs genannten Branchen stellen insgesamt 57,8\% der Arbeitsplätze.

Innerhalb der Sektoren weist der tertiäre Sektor mit 32\% einen viermal höheren Anteil der Teilzeitbeschäftigten auf als der sekundäre Sektor $(9 \%)$. Dies dürfte vor allem auf die verschiedenen Arbeitsabläufe und betriebsinternen Bedürfnisse innerhalb der Sektoren sowie die unterschiedlichen Möglichkeiten zur Flexibilisierung der Beschäftigungsverhältnisse zurückzuführen sein. Unter den sechzehn vom Beschäftigungsvolumen bedeutendsten Branchen nehmen, gemessen am Anteil der Teilzeitstellen, die chemische Industrie $(8,4 \%)$, der Maschinenbau $(8,0 \%)$ und das Baugewerbe $(6,0 \%)$ nur die Ränge 14 bis 16 ein (Abb. 1). Hohe Anteile an Teilzeitbeschäftigten weisen dagegen das Unterrichtswesen $(58,4 \%)$, das Gesundheits- und Sozialwesen (48,8\%), der Bereich Nachrichtenübermittlung $(45,4 \%)$ und der Einzelhandel $(38,9 \%)$ auf. In diesen arbeitsintensiven, überwiegend personenbezogenen und häufig durch Frauen abgedeckten Tätigkeitsfeldern eröffnen Teilzeitbeschäftigung sowie die zeitliche Befristung von Arbeitsverträgen und die Zeitarbeit unterschiedliche Möglichkeiten der Kostenreduktion. Dazu gehören niedrigere Löhne, kaum Urlaubsanspruch, kürzere Kündigungsfristen und flexiblerer Arbeitseinsatz (RIFKIN 1996: 135, 148; KRÄTKE 1996: 70).

\subsection{Arbeitsstättenstruktur}

Die Betriebsgrössenstruktur des Untersuchungsgebietes wird massgeblich von Klein- und Mittelbetrieben mit weniger als 100 Beschäftigten geprägt. So weisen $79,1 \%$ oder 13633 Arbeitsstätten weniger als 10 Beschäftigte auf, und etwa jede vierte Arbeitsstätte verfügt lediglich über einen Beschäftigten. Auf die Grössenklasse mit mehr als 200 Beschäftigten entfallen hingegen nur $0,8 \%$ der Arbeitsstätten. Nach Sektoren differenziert zeigt sich, dass der Anteil der Betriebe mit weniger als $10 \mathrm{Be}$ schäftigten im II. Sektor $(71,2 \%)$ geringer ist als im III. Sektor $(80,9 \%)$. Demgegenüber ist der Anteil der Arbeitsstätten mit mehr als 49 Beschäftigten im sekundären Sektor (6,9\%) doppelt so hoch wie im tertiären Bereich (3,5\%).

Zu den «grossbetrieblich» strukturierten Branchen (Betriebe mit mehr als 100 Beschäftigten) gehören u. a. die chemische Industrie $(22,5 \%)$, die Nachrichtenübermittlung $(10,4 \%)$, das Kreditgewerbe $(8,2 \%)$, der Maschinenbau (7,9\%) sowie das Versicherungsgewerbe $(6,6 \%)$. Durch ausgeprägt kleinbetriebliche Strukturen (1-9 Beschäftigte) sind hingegen die unternehmensorientierten Dienstleistungen $(88,5 \%)$, der Einzelhandel $(85,0 \%)$ und das Gesundheits- und Sozialwesen $(83,3 \%)$ gekennzeichnet.

\section{Wandel der regionalen Wirtschaftsstruktur zwischen 1991 und 1995}

\subsection{Beschäftigung und Arbeitslosigkeit}

Zwischen 1991 und 1995 ging die Gesamtbeschäftigung im Untersuchungsgebiet um $-6,9 \%$ oder 17323 Arbeitsplätze zurück. Die Stadt Basel ist mit $-7,8 \%$ stärker von Arbeitsplatzverlusten betroffen als das Umland mit $-5,1 \%$. Infolge der Flexibilisierung einerseits und der zunehmenden Unternehmenskonzentration (Fusionen) andererseits werden zudem zunehmend gut bezahlte Arbeitsplätze für qualifizierte Mitarbeiter abgebaut. Parallel dazu stieg die Zahl der Arbeitslosen im Kanton Basel Stadt von 1,7\% auf $4,9 \%$ und im Bezirk Arlesheim (als Bezugswert für die ausgewählten Umlandgemeinden) von $0,5 \%$ auf $1,3 \%$. Die Folgewirkungen sind folglich für Stadt und Umland unterschiedlich stark.

\subsection{Intersektoraler Bedeutungswandel}

Im sekundären Sektor wurden deutlich mehr Arbeitsplätze abgebaut $(-15,3 \%$ oder -13600$)$ als im tertiären Sektor $(-2,3 \%$ oder -3327$)$, was auf dessen grösseres Rationalisierungspotential zurückzuführen ist. Dadurch sank der Anteil des II. Sektors an der Gesamtbeschäftigung um weitere 3,2\% auf $32,2 \%$. Vom Beschäftigtenrückgang betroffen 
waren vor allem die durch Massenproduktion gekennzeichneten Branchen (chemische Industrie: $-20,7 \%$, Maschinenbau: $-11,6 \%$ ) sowie das Baugewerbe $(-8,3 \%)$. Ursachen sind die Konzentration auf das Kerngeschäft, die Verringerung der Fertigungstiefe und die Auslagerung von Unternehmensaktivitäten.

Der tertiäre Sektor konnte seine anteilsmässige Bedeutung ausbauen, obwohl es nicht zu einer absoluten Zunahme der Beschäftigung kam. Von hohen Beschäftigtenverlusten betroffen sind die Vermietung beweglicher Sachen ohne Bedienungspersonal (Maschinen und Geräte aller Art) mit $-36,6 \%$, der Bereich Unterhaltung, Kultur und Sport $(-26,0 \%)$ sowie das Versicherungsgewerbe $(-13,9 \%)$. Eine «Verlagerung» von Arbeitsplätzen zugunsten des III. Sektors wird somit deutlich. Dieser profitiert neben dem Outsourcing vom steigenden Bedarf an Forschung und Entwicklung (F\&E), von der räumlichen Streuung der Produktionsanlagen im Rahmen der Globalisierung und der Produktedifferenzierung, die eine immer stärkere Spezialisierung erfordert (SASSEN 1996: 76).

\subsection{Intrasektorale Umstrukturierungen}

Auch innerhalb der Sektoren kam es zu Umstrukturierungen. Im tertiären Sektor ist eine Verlagerung der Beschäftigtenanteile zugunsten der höherwertigen Dienstleistungen zu beobachten (Abb. 2). Hohe Beschäftigtenzuwächse verzeichnen insbesondere mit dem Kredit- und Versicherungsgewerbe verbundene Tätigkeiten $(+60,5 \%)$, der F \& E-Bereich $(+39,2 \%)$, die Informatikdienste $(+35,4 \%)$, die Nachrichtenübermittlung $(+30,1 \%)$ sowie die Hilfs- und Nebentätigkeiten für den Verkehr $(+24,3 \%)$. Da es sich hierbei primär um produktions- und nicht um konsumorientierte Dienstleistungen handelt, bleibt dieser Wirtschaftsbereich stark abhängig von der Entwicklung des sekundären Sektors. Ursachen dieser Umstrukturierung sind das verstärkte Outsourcing sowie der allgemeine Wandel zur Wissensgesellschaft, in der Kommunikation, Informatik und Forschung eine immer grössere Bedeutung einnehmen. Demgegenüber stehen Rationalisierungen in traditionellen Bereichen des III. Sektors, wie dem Versicherungs- oder Kredit-

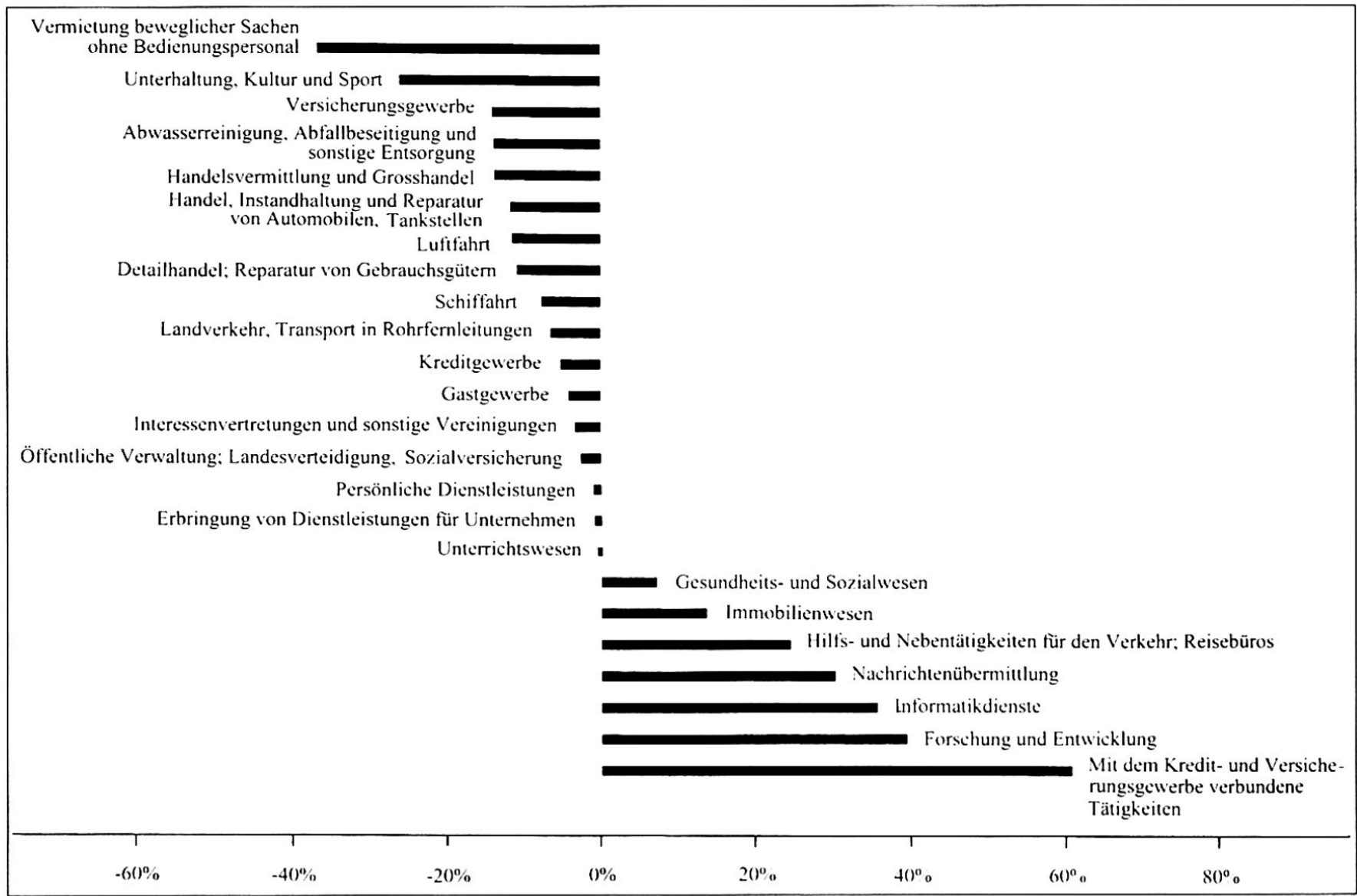

Abb. 2: Entwicklung der Beschäftigung in den Branchen des tertiären Sektors 1991-1995

Employment in the branches of the third sector 1991-1995

Evolution des emplois dans les branches du secteur tertiaire 1991-1995

Quelle: Statistisches Amt Des Kantons Basel-Landschaft [Hrsg.] (1997): Betriebszählung 1995. 
gewerbe, die eine Abnahme von $-13 \%$ bzw. $-5 \%$ aufweisen. Beide Branchen sind, abgesehen von der allgemeinen Marktsättigung, verstärkt von den Auswirkungen der Globalisierung betroffen.

Innerhalb des sekundären Sektors sind lediglich drei Branchen durch eine Beschäftigungszunahme gekennzeichnet. Hierzu zählen die dem HighTech-Bereich zuzuordnende Herstellung von medizinischen Geräten und Präzisionsinstrumenten $(+15,5)$, das Papier- und Kartongewerbe $(+23,4)$ sowie die Gewinnung von Steinen und Erden $(+74,3 \%)$, deren Wachstum die geringe Ausgangsbasis jedoch relativiert. Alle übrigen industriellen Branchen, insbesondere die traditionellen Wachstumsbranchen, weisen dagegen erhebliche Beschäftigtenverluste auf. Dies und die Tatsache, dass der durchschnittliche Beschäftigtenrückgang im sekundären Sektor mit $-15,3 \%$ deutlich höher ausfällt als im Dienstleistungssektor $(-2,3 \%)$, bestätigen wiederum die These der Bedeutungsverlagerung zum III. Wirtschaftssektor.

\subsection{Interne Flexibilisierung}

Vom Arbeitsplatzabbau sind primär Vollzeitarbeitsplätze betroffen (-9,3\% oder 18005 Stellen). Auch hier weist der sekundäre Sektor mit einem Rückgang der Vollzeitstellen um $-15,8 \%$ deutlich höhere Verluste auf als der tertiäre Sektor $(-4,6 \%)$. Umgekehrt ist die Anzahl der Teilzeitarbeitsplätze zwischen 1991 und 1995 um insgesamt 1,2\% oder 682 Teilzeitstellen gestiegen. Dahinter verbirgt sich ein Verlust von -768 Teilzeitarbeitsplätzen im sekundären Sektor und ein entsprechender Gewinn von 1450 Stellen im III. Sektor.

Während sich das Verhältnis von Vollzeit- zu Teilzeitarbeitsplätzen innerhalb der Sektoren praktisch nicht verschoben hat, kann insgesamt ein Anstieg der Teilzeitstellen um $2 \%$ auf $24,9 \%$ festgestellt werden. Das höchste Wachstum an Teilzeitstellen weisen die Nachrichtenübermittlung $(+25,0 \%)$ und das Gesundheits- und Sozialwesen $(+5,9 \%)$ auf. Im ganzen ist eine Zunahme der Teilzeitarbeit, insbesondere in den dafür prädestinierten Branchen des tertiären Sektors, zu beobachten.

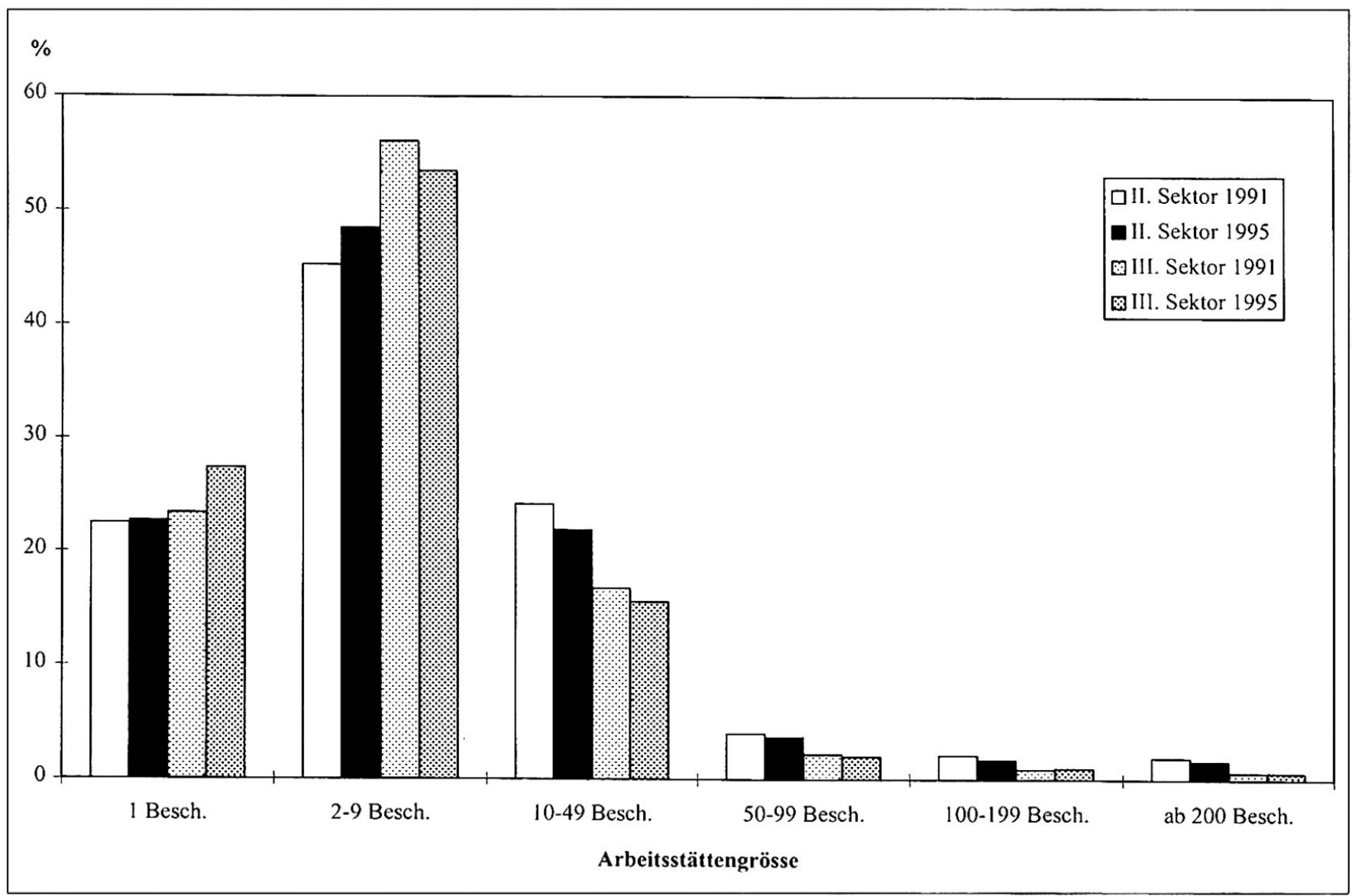

Abb. 3: Arbeitsstättengrößen im sekundären und tertiären Sektor 1991 und 1995

Enterprises in the second and the third sectors 1991 and 1995, by size class

Taille des établissements dans le secteur secondaire et dans le secteur tertiaire 1991 et 1995

Quelle: Statistisches Amt Des Kantons Basel-Landschaft [Hrsg.] (1997): Betriebszählung 1995. 


\subsection{Umstrukturierung der Betriebsgrössen}

Die Betrachtung der Betriebsgrössenklassen in den Sektoren II und III zeigt einen prozentualen Zuwachs der Arbeitsstätten mit nur einem Beschäftigten von $23,3 \%$ auf $26,5 \%$. Dieser Zunahme steht eine Abnahme in sämtlichen anderen Klassen gegenüber. Der stärkste Rückgang fand mit $-1,5 \%$ im Bereich der Arbeitsstätten mit 2 bis 9 Beschäftigten statt. Es kann also nicht von einem Bedeutungsgewinn der Klein- und Mittelbetriebe gesprochen werden. Vielmehr ist eine Gründerwelle zu beobachten, die nicht zuletzt mit dem Übergang eines Teils der freigesetzten Beschäftigten in die Selbständigkeit zusammenhängt. Zudem ist das Wachstum der KMUs als eine Folge des Outsourcings anzusehen, das nicht ursächlich zu einem Beschäftigtenzuwachs führt.

Die sektorale Differenzierung zeigt die Hauptzunahme im sekundären Sektor im Bereich der Betriebe mit 2 bis 9 Beschäftigten ( $+3,2 \%$ auf $48,5 \%)$. Bei den Dienstleistungen erfolgte das Hauptwachstum dagegen im Segment der Arbeitsstätten mit einem Beschäftigten. Hier erhöhte sich der Anteil der Arbeitsstätten von 23,3\% auf 26,5\% (Abb.3). Das Wachstum konzentriert sich somit auf Klein- und Kleinstbetriebe und weniger auf mittelständische Unternehmen. Die kritische Betriebsgrösse ist im sekundären Sektor höher als im tertiären. Insgesamt ist der Weg in die Selbständigkeit im Dienstleistungsbereich, vor allem auch durch die modernen Kommunikationstechnologien, leichter. Im industriellen Sektor sind die zur Betriebsgründung notwendigen Investitionen höher, und es entstehen meist nicht unerhebliche Transportkosten.

\section{Räumliche Restrukturierungsprozesse in ausgewählten Branchen}

Für den im Folgenden analysierten Bedeutungswandel einzelner Branchen (Karten 1-5) wurde die Differenz zwischen den Anteilszahlen der Beschäftigten je Branche und Gemeinde von 1995 und 1991 bestimmt.

\subsection{Chemische Industrie}

Der Beschäftigungsschwerpunkt in der chemischen Industrie lag 1991 mit einem Arbeitsplatzanteil von $76,9 \%$ in der Stadt Basel. Entsprechend entfielen auf die Umlandgemeinden nur 7951 Stellen (23,1\%) in dieser Branche. 1995 haben sich die Anteilswerte weiter zugunsten des baselstädtischen Standortes $(+2,3 \%)$ verschoben, der nunmehr 79,2\% der Arbeitsplätze in der Chemie stellt. Hintergrund dieser Entwicklung ist ein massiver Stellenabbau, der im Umland mit

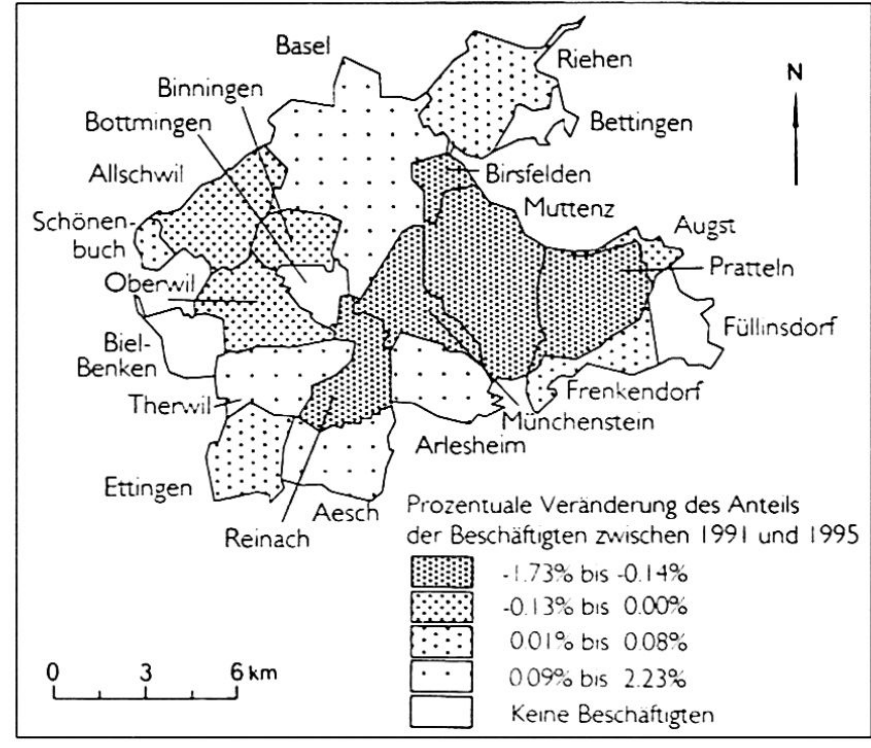

Karte 1: Bedeutungswandel der chemischen Industrie 1991-1995 Change in the relative importance of the chemical industry 1991-1995 Changement de l'importance de l'industrie chimique 1991-1995 Quelle: Statistisches Amt Des Kantons BaselLANDSCHAFT [Hrsg.] (1997): Betriebszählung 1995.

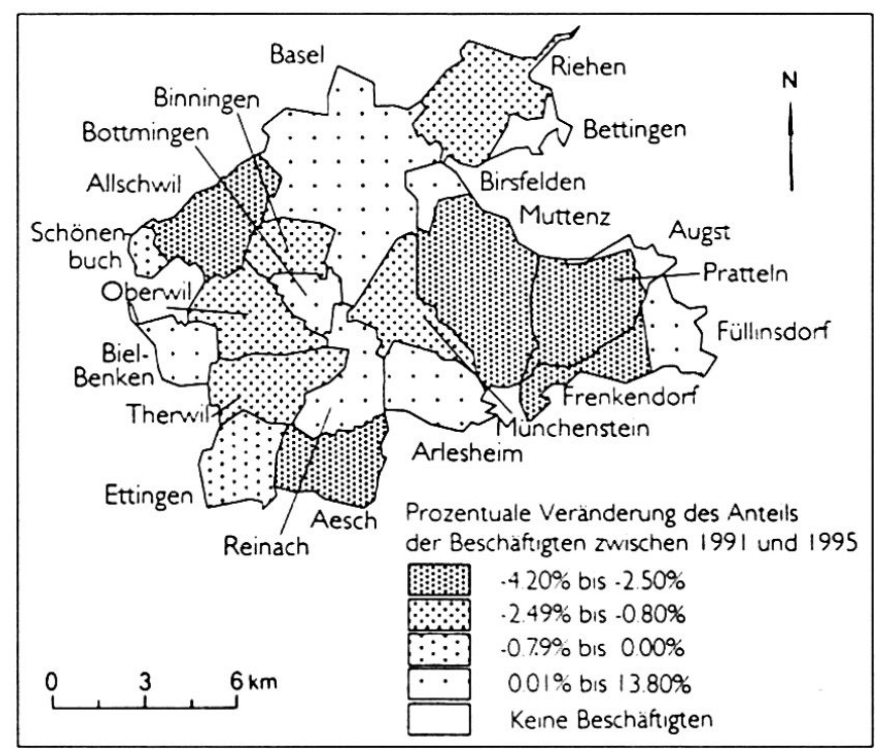

Karte 2: Bedeutungswandel des Maschinenbaus 1991-1995 Change in the relative importance of the machine and tool industry 1991-1995 Changement de l'importance de la construction de machines 1991-1995 Quelle: Statistisches Amt Des Kantons BaselLANDSCHAFT [Hrsg.] (1997): Betriebszählung 1995. 


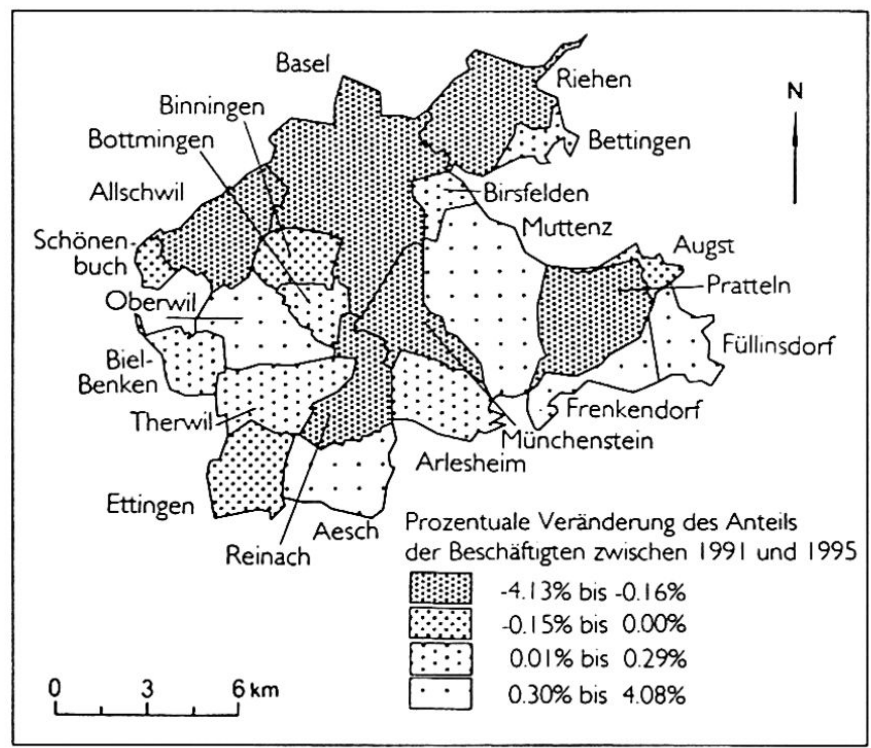

Karte 3: Bedeutungswandel

des Detailhandels 1991-1995

Change in the relative importance of

retail trade 1991-1995

Changement de l'importance

du commerce de détail 1991-1995

Quelle: Statistisches Amt Des Kantons Basel-

LANDSCHAFT [Hrsg.] (1997): Betriebszählung 1995.

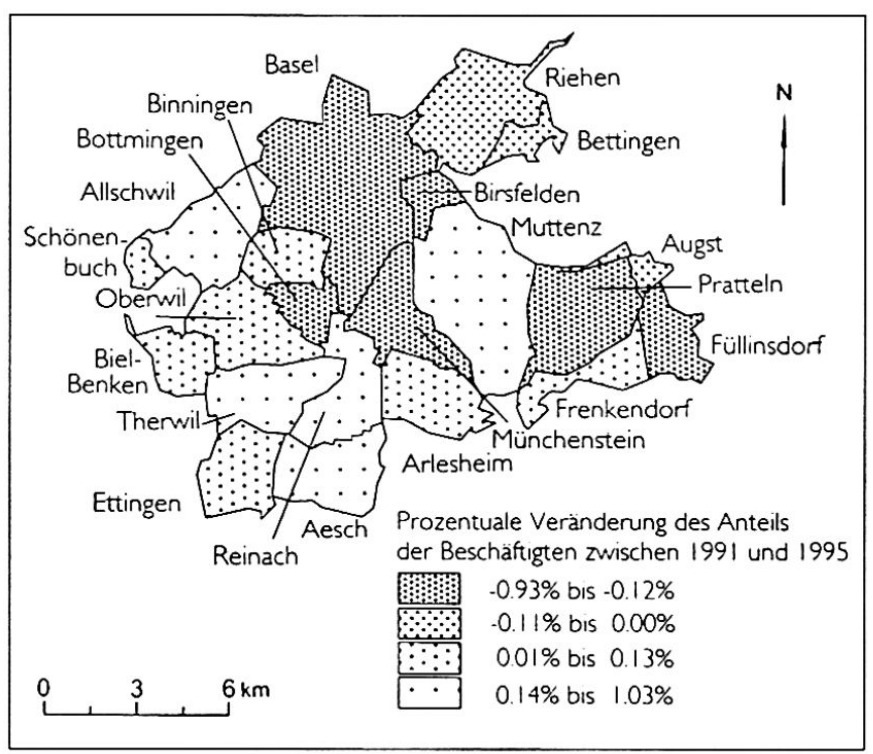

Karte 5: Bedeutungswandel der unternehmensorientierten Dienstleistungen 1991-1995 Change in the relative importance of business services 1991-1995

Changement de l'importance

d'autres services fournis aux entreprises 1991-1995 Quelle: Statistisches Amt Des Kantons BaselLANDSCHAFT [Hrsg.] (1997): Betriebszählung 1995.

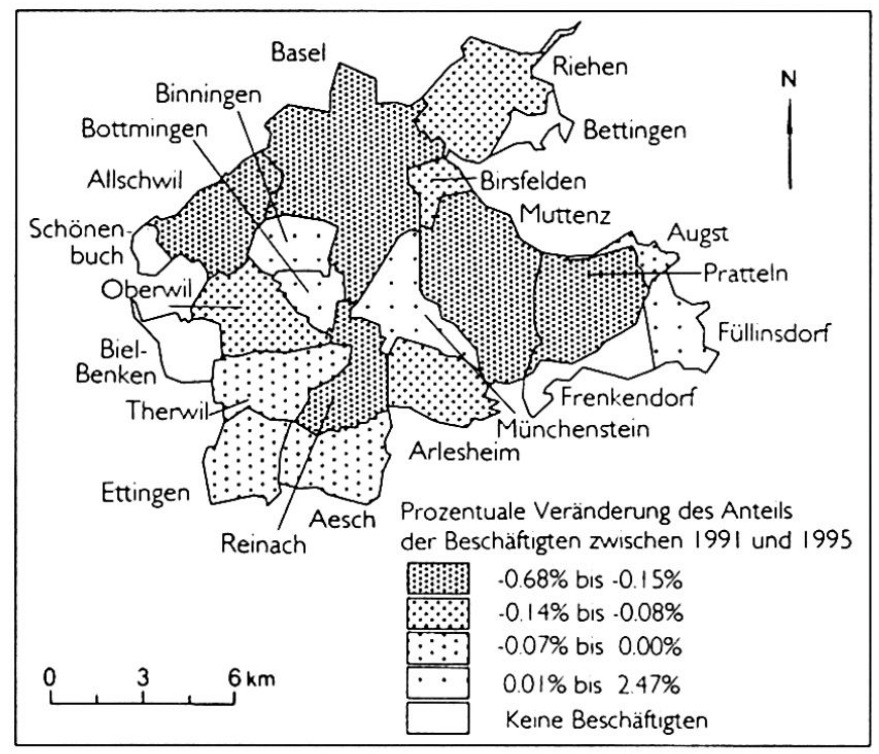

Karte 4: Bedeutungswandel des Kreditgewerbes 1991-1995 Change in the relative importance of finance banking 1991-1995

Changement de l'importance de l'intermédiation financière 1991-1995

Quelle: Statistisches Amt Des Kantons BaselLANDSCHAFT [Hrsg.] (1997): Betriebszählung 1995. einem Rückgang der Beschäftigten um 28,7\% jedoch deutlich stärker ausfiel als in der Stadt Basel $(-18,3 \%)$ (Tab. 1). Von einer räumlichen Dekonzentration in das Umland kann folglich nicht gesprochen werden.

Das räumliche Standortmuster lässt eine Konzentration der chemischen Industrie entlang einer dem Rhein folgenden Achse mit den Gemeinden Muttenz und Pratteln erkennen. Standortfaktor ist die Nähe zum Rhein. Die prozentual grössten Bedeutungsverluste treten in diesem traditionellen Standortbereich der Chemie sowie in den Gemeinden Münchenstein und Reinach auf (Karte 1). Die geringsten Abnahmen sowie leichte Zuwächse verzeichnen die peripher im Untersuchungsgebiet gelegenen Gemeinden.

Ursache des Beschäftigtenabbaus (1991-1995: $-20,7 \%$ ) sind u.a. Rationalisierungsmassnahmen im Vorfeld der Fusion von Ciba-Geigy und Sandoz sowie verstärktes Outsourcing im Bereich Informatik, handwerklicher Unterhalt, Werksicherheit, usw. (FüEg 1998: 22 f.). Der anteilsmässige Bedeutungsgewinn der Stadt Basel in der Chemie dürfte vor allem den Ausbau von Forschung und Entwicklung sowie von Headquarterfunktionen widerspiegeln. Bedeutungsverluste in den traditio- 


\begin{tabular}{llrrrr}
\hline & & \multicolumn{2}{c}{1991} & \multicolumn{2}{c}{1995} \\
Branche & & absolut & $\%$ & absolut & $\%$ \\
Chemische & Stadt & 24489 & 76,9 & 21639 & 79,2 \\
Industrie & Umland & 7951 & 23,1 & 5667 & 20,8 \\
& & & & & \\
Einzelhandel & Stadt & 15936 & 65,5 & 13344 & 61,4 \\
& Umland & 8394 & 34,5 & 8400 & 38,6 \\
unternehmens- & & & & & \\
bezog. Dienst- & Stadt & 13746 & 72,9 & 13545 & 72,4 \\
leistungen & Umland & 5117 & 27,1 & 5171 & 27,6 \\
& & & & & \\
Kreditgewerbe & Stadt & 8797 & 91,8 & 8295 & 91,1 \\
& Umland & 783 & 8,2 & 806 & 8,9 \\
& & & & & \\
Maschinenbau & Stadt & 1089 & 24,7 & 1157 & 29,7 \\
& Umland & 3324 & 75,3 & 2745 & 70,3 \\
\hline
\end{tabular}

Tab. 1: Entwicklung der Beschäftigtenzahlen in ausgewählten Branchen 1991-1995

Employment in selected branches 1991-1995

Changement des emplois

en branches choisies entre 1991 et 1995

Quelle: Statistisches Amt Des Kantons Basel-

LANDSCHAFT [Hrsg.] (1997): Betriebszählung 1995.

nellen Chemiestandorten im Umland können auf die zunehmende Verlagerung der Produktion ins Ausland zurückgeführt werden.

Um die Hypothese der Dekonzentration genauer zu untersuchen, müsste eine weitere Differenzierung nach den dispositiven und operativen Unternehmensfunktionen erfolgen. Bedeutungsgewinne der Chemie in den entfernteren Umlandgemeinden können dadurch erklärt werden, dass infolge der Ausgliederung von Geschäftstätigkeiten zunehmend auch hochqualifizierte Mitarbeiter freigesetzt werden (FüEg 1998: 21), die wiederum neue kleinere Unternehmen in Bereichen mit höherer Wohn- und Umweltqualität gründen. So verzeichneten die Gemeinden Riehen, Aesch, Arlesheim, Schönenbuch und Therwil im Untersuchungszeitraum (geringe) Beschäftigungszunahmen in der Chemie.

\subsection{Maschinenbau}

Im Maschinenbau lag der Beschäftigungsschwerpunkt eindeutig im Umland (1991: 75,3\% der Beschäftigten im Umland, 1089 Personen oder 24,7\% in der Kernstadt). Aufgrund des Arbeitsplatzwachstums in der Stadt (+1157 Stellen) und eines mehr als doppelt so hohen Beschäftigungsabbaus in den Umlandgemeinden konnte Basel seinen Beschäftigtenanteil jedoch auf $29,7 \%$ (1995) steigern (Tab. 1). Im Maschinenbau ist somit keine fortschreitende räumliche Dekonzentration der Beschäftigten festzustellen.
Nach Gemeinden differenziert, weisen Bottmingen, Reinach, Aesch sowie Muttenz und Pratteln die höchsten Beschäftigtenanteile im Maschinenbau auf. Neben der Stadt Basel konnten insbesondere Bottmingen und Reinach, aber auch Füllinsdorf Bedeutungsgewinne verzeichnen (Karte 2). Sie verfügen über relativ grosse Flächenreserven für eine Expansion dieser Branche. Starke Abnahmen sind dagegen in den eher traditionellen Standorten Muttenz, Pratteln, Frenkendorf sowie in Allschwil zu beobachten. Hier sind die Reserven an Gewerbeflächen relativ gering.

\subsection{Detailhandel und Reparatur von Gebrauchsguitern}

Den höchsten Beschäftigtenanteil weist die Stadt Basel auf. Bereits 1991 entfielen jedoch schon mehr als ein Drittel der Arbeitsplätze auf die Umlandgemeinden $(34,5 \%, 8394$ Stellen). Obwohl die Beschäftigtenzahl bis 1995 im Umland stagnierte, konnte dieses aufgrund des starken Arbeitsplatzabbaus in der Kernstadt $(-2592 \mathrm{Be}-$ schäftigte) seine relative Bedeutung um $4,1 \%$ steigern (Tab. 1). Somit ist eine deutliche Bedeutungsverlagerung in das Umland festzustellen. Die Beschäftigtenabnahme im städtischen Einzelhandel hängt mit dem Suburbanisierungsprozess zusammen, der Einzelhandel folgt der abwandernden Bevölkerung. Autogerechte grossflächige Einzelhandelseinrichtungen auf der «grünen Wiese» (Einkaufszentren, Fachmärkte, Verbrauchermärkte) sowie niedrigere Bodenpreise führen zu einem weiteren Bedeutungsgewinn des Einzelhandels im Umland.

Zwischen der Bevölkerungs- und der Einzelhandelsentwicklung in den einzelnen Gemeinden (Karte 3) ist ein Zusammenhang festzustellen. Die durch Bevölkerungs- bzw. Wanderungsverluste gekennzeichneten Gemeinden Allschwil, Arlesheim, Münchenstein und Pratteln weisen die höchsten Bedeutungsverluste im Einzelhandel auf. Umgekehrt sind die Gemeinden Oberwil, Aesch, Frenkendorf und Füllinsdorf mit einer stagnierenden bis positiven Bevölkerungsentwicklung durch Bedeutungsgewinne charakterisiert (Schneider-Sliwa, Kampschulte, Nommel, SandtNER, Strassmann \& WafFensChmidT 1999). Eine Ausnahme bildet hier lediglich die Gemeinde Muttenz. Da die Suburbanisierung anhält und zunehmend in den ländlichen Raum vordringt, ist aber auch im engeren Umland Basels zukünftig mit einem Abbau der Einzelhandelsbeschäftigten zu rechnen, d.h., der Dekonzentrationsprozess wird sich noch weiter fortsetzen. 


\subsection{Kreditgewerbe}

Kennzeichen des Kreditwesens ist die hohe Konzentration der Beschäftigten in der Stadt Basel sowie die geringe Dynamik der Branche. Während 1991 91,8\% der im Untersuchungsgebiet im Kreditgewerbe Tätigen in der Kernstadt arbeiteten, nahm dieser Anteil bis 1995 um 0,7\% zugunsten des Umlandes ab. Dahinter verbirgt sich eine absolute Abnahme um 502 Arbeitsplätze in der Stadt bei einem gleichzeitigen Stellenzuwachs von 23 auf insgesamt 806 Stellen in den Umlandgemeinden (Tab. 1). Somit sind weder weiter fortschreitende Konzentrations- noch Dekonzentrationsprozesse festzustellen.

Abgesehen von der Dominanz in der Stadt Basel, ist das Kreditgewerbe nur noch in den Gemeinden Münchenstein, Reinach und Binningen von grösserer Bedeutung. Mit Ausnahme von Reinach sind dies auch die Standorte, die einen entsprechenden Bedeutungsgewinn verzeichnen (Karte 4). Münchenstein ist zugleich die einzige Gemeinde, in der auch absolut gesehen die Zahl der Arbeitsplätze in dieser Branche von 23 auf 247 Stellen gestiegen ist. Verluste weisen dagegen neben Basel die Gemeinden mit rückläufiger Bevölkerungsentwicklung (Allschwil, Muttenz, Pratteln, Reinach) auf. Weitere Ursachen dürften der Abbau überflüssiger Kapazitäten (Overbanking) sowie die Verlagerung von arbeitsaufwendigen Funktionen zum Konsumenten (Geldbezug, Telebanking) darstellen.

Für die weitere Entwicklung des Finanzbereiches am Standort Basel (Stadt und Umland) ist auch die Stellung Basels im schweizerischen Städtesystem von Relevanz. Nach SASSEN (1996: 123 ff.) findet die Konzentration von Finanzaktivitäten jeweils in einem einzigen Zentrum, im Fall der Schweiz in Zürich, statt. Es vollzieht sich ein grossräumigerer Prozess der selektiven Dekonzentration (aus Basler Sicht) bzw. Konzentration (aus Zürcher Sicht) von Funktionen. Die Bedeutungsabnahme Basels als «Bankenstandort» spiegelt sich bereits in entsprechenden Arbeitsstättenverlusten (1991 bis 1995: -5,0\% ) wider, die sich u.a. im Zuge der Fusion von UBS und Schweizer Bankverein noch verstärken werden. Vom Personalabbau betroffen sind dabei vor allem Grossbanken, während kleine und mittlere Banken z.T. von dieser Umstrukturierung profitieren (FÜEG 1998: 30 f.).

\subsection{Erbringung von Dienstleistungen für Unternehmen}

Die unternehmensnahen Dienstleistungen weisen den insgesamt geringsten Beschäftigtenrückgang $(-0,8 \%)$ auf. Differenziert nach Stadt und Umland fand dieser Abbau in der Kernstadt statt, die 201 Arbeitsplätze verlor, während die Umlandgemeinden insgesamt einen Zuwachs von 54 Stellen verzeichnen konnten. Anteilsmässig bedeutet dies eine Verschiebung um $0,5 \%$ auf $27,6 \%$ zugunsten des Umlandes (Tab. 1). Entgegen der Ausgangshypothese zeichnet sich damit auch im Bereich der Dienstleistungen für Unternehmen kein fortschreitender Konzentrationsprozess der Beschäftigten in der Kernstadt ab.

Innerhalb des Umlandes stehen den Gemeinden Birsfelden, Bottmingen, Münchenstein, Pratteln und Füllinsdorf mit einem Bedeutungsverlust der Branche von $-0,1 \%$ bis $-0,9 \%$ die Gemeinden Muttenz, Allschwil, Reinach, Therwil und Aesch mit dem höchsten Bedeutungszuwachs von $+0,1 \%$ bis $+1 \%$ (Karte 5 ) gegenüber. Auch hier ist die Entwicklung nicht homogen. Ein Vergleich der räumlichen Branchenstrukturen weist Gemeinden mit den höchsten Bedeutungsgewinnen im unternehmensorientierten Dienstleistungsbereich als jene mit den stärksten Bedeutungsverlusten im sekundären Sektor (Karte 1 und 2) aus. Dies legt die Vermutung nahe, dass bei den hier ansässigen industriellen «Grossbetrieben» zumindest zum Teil eine Auslagerung von Dienstleistungsfunktionen stattgefunden hat.

Dem theoretisch angenommenen Konzentrationsprozess wirkt möglicherweise entgegen, dass es sich hier vermehrt um Kleinbetriebe handelt, die durch das Outsourcing und die Freisetzung höher qualifizierter Arbeitnehmer entstanden sind. Die nachfolgenden Jungunternehmer bevorzugen $u$. U. eine teilgewerbliche Nutzung der eigenen Wohnung bzw. Betriebsstandorte in der Nähe des Wohnsitzes und versuchen Kostenvorteile durch die im Umland niedrigeren Miet- und Bodenpreise auszuschöpfen. Sowohl die räumliche Nähe zur Stadt Basel als auch die gegebenen Telekommunikationsmöglichkeiten sichern auch von diesen dezentraleren Standorten aus den notwendigen Kontakt zum Kunden.

\section{Ausblick: Räumliche Arbeitsteilung zwischen Stadt und Umland}

Die Ergebnisse bestätigen, dass sich weltweit zu beobachtende wirtschaftliche Restrukturierungsprozesse in ähnlicher Form auch in Basel vollziehen und zur Veränderung des Wirtschaftsraumes beitragen. Analog zu den aufgestellten Arbeitshypothesen ist die Entwicklung gekennzeichnet durch:

- rückläufige Beschäftigtenzahlen in fast allen Wirtschaftsbereichen, insbesondere aber im sekundären Sektor

- steigende Arbeitslosigkeit

- Umstrukturierungen innerhalb der Sektoren durch verstärkten Beschäftigtenabbau in den 
Branchen mit standardisierter Massenproduktion und Ausbau der unternehmensorientierten Dienstleistungen

- fortschreitende Tertiärisierung

- flexiblere Beschäftigungsverhältnisse mit der Tendenz zur Teilzeitbeschäftigung

- Veränderungen in den Unternehmensstrukturen: einer Vergrösserung der Unternehmenskonzerne durch Fusionen (Chemie, Banken) steht eine Verringerung der Betriebsgrössenstrukturen zugunsten flexiblerer, kleiner Betriebseinheiten gegenüber

- Eine Gründungswelle von Einpersonenunternehmen im Dienstleistungssektor.

Prozesse der räumlichen Konzentration und Dekonzentration bestimmter wirtschaftlicher Funktionen, die sich in Global Cities oder in Grossstadtregionen vollziehen, sind im Stadt-Umland-Bereich Basel dagegen nur bedingt zu erkennen. Ursachen hierfür dürften in der vergleichsweise «geringen» Grösse der Agglomeration und regionsspezifischen Besonderheiten wie der Dominanz der chemischen Industrie oder der exponierten Grenzlage begründet sein. Darüber hinaus werden bestimmte Entwicklungen wesentlich durch Polarisierungen im nationalen Städtesystem beeinflusst.

Zusätzlich sind Stadt und Umland hinsichtlich der Art und Intensität der Auswirkungen unterschiedlich von den beschriebenen Entwicklungen betroffen. Insgesamt kann festgehalten werden, dass die räumliche Arbeitsteilung zwischen Stadt und Umland dadurch weiter vorangetrieben wird und dass sich verstärkte Polarisierungen innerhalb des Umlandes abzeichnen (ADAM \& BLACH 1996: $187 \mathrm{ff}$.). Während die Stadt Arbeitsplätze und Infrastruktur für das Umland bereitstellt, bietet letzteres erweiterungsfähige Betriebsflächen und Erholungsmöglichkeiten für die Stadt. Einseitige Belastungen entstehen zudem durch den steigenden Pendelverkehr in die Stadt sowie den zunehmenden Flächenverbrauch im Umland. Um diesen Entwicklungen entgegenzuwirken und die Wettbewerbsfähigkeit der Region als Ganzes (KIEPE 1996: 307) zu sichern, erscheint es dringend notwendig, entsprechende Ausgleichsmechanismen zu schaffen. Voraussetzung hierfür ist die Kommunikations- und Kooperationsbereitschaft der Gebietskörperschaften und der wirtschaftlichen Akteure in der Region. Insgesamt bedarf es eines Gesamtkonzeptes, das notwendige Einzelmassnahmen unter Berücksichtigung ökonomischer, ökologischer und sozialer Belange inhaltlich und räumlich koordiniert. Als solche können aufgeführt werden:

- Massnahmen des interkantonalen bzw. interkommunalen Finanzausgleichs
- restriktive Ausweisung von Gewerbeflächen und Bauland im Umland

- Beschränkungen hinsichtlich der Grösse und Anzahl grossflächiger Einzelhandelseinrichtungen auf der «grünen Wiese»

- Massnahmen zur Steigerung der Attraktivität der Kernstadt als Wohn- und Lebensraum

- verstärkte Förderung des öffentlichen Verkehrs zur Verminderung des Gesamtverkehrsaufkommens in der Stadt

- Verbesserung der Umweltqualität und der Erholungsfunktion in der Kernstadt u.a. durch Entwicklung eines umfassenden Grünflächenkonzeptes.

Problematisch ist jedoch, dass sich im allgemeinen immer mehr raumbedeutsame Entscheidungen externer Kontrolle bzw. lenkender Eingriffe entziehen. "Speziell den Grossunternehmen ermöglicht die weiter zunehmende Kapitalkonzentration eine regionsübergreifende Regulation von Kapitalbewegungen, Produktions- und Absatzbeziehungen, die sie im Rahmen ihrer multiregional verteilten Unternehmensabteilungen intern steuern. Damit können sich diese Unternehmen einem regulierenden Eingriff von seiten nationalstaatlicher Institutionen immer besser entziehen, und die Entwicklungs-Chancen ganzer Regionen und Städte unternehmensintern bestimmen» (KRÄTKE 1996: 18).

Für eine nachhaltige Lösung der aus der disparitären Raumnutzung resultierenden Probleme ist es unabdingbar, langfristig ökologische und soziale Folgekosten stärker in den wirtschaftlichen Entscheidungsprozess mit einzubeziehen.

\section{Literatur}

Adam, B. \& A. Blach (1996): Räumliche Arbeitsteilung in Grossstadtregionen - interkommunale und raumordnerische Konfliktkategorien. - In: Informationen zur Raumentwicklung 4/5: 187-208.

FÜEG, R. (Hrsg.) (1998): Wirtschaftsstudie Nordwestschweiz 1996/97. - = Schriften der Regio 7.19, Basel. KiEPE, F. (1996): Die Stadt und ihr Umland. Zur Notwendigkeit der Bildung von Stadtregionen. - In: Informationen zur Raumentwicklung, 4/5: 307-315. KRÄTKE, S. (1996): Stadt-Raum-Ökonomie: Einführung in aktuelle Problemfelder der Stadtökonomie und Wirtschaftsgeographie. $-=$ Stadtforschung aktuell 53, Basel.

LESER, H. (Hrsg.) (1997): Diercke-Wörterbuch Allgemeine Geographie. - Braunschweig.

Lutz, C. (1997): Der Arbeitnehmer ist tot - es lebe die Lebensunternehmerin! - In: $\mathrm{HENSCH}, \mathrm{CH}$. \& U. WISMER (Hrsg.): Zukunft der Arbeit, Stuttgart: 129-135. 
Rifkin, J. (1996): Das Ende der Arbeit und ihre Zukunft. - Frankfurt/Main.

SASSEN, S. (1996): Metropolen des Weltmarkts. Die neue Rolle der Global Cities. - Frankfurt/Main.

Schneider-Sliwa, R., Kampschulte, A., Nommel, J.U., Sandtner, M., Strassmann, R. \& Ch. WaffenSCHMIDT (1999): Bevölkerungsstrukturen und Bevölkerungsdynamik beider Basel. - = Stadt und Region 1, Basel.

Statistisches Amt Des Kantons Basel-LandSCHAFT (Hrsg.) (1997): Betriebszählung 1995. Ergebnisse für den 2. und 3. Sektor. Liestal.

\section{Zusammenfassung: Restrukturierungsprozesse einer Stadt-Umland-Ökonomie:

Die Region Basel ist von Globalisierungstendenzen betroffen, die zu Restrukturierungsprozessen der Wirtschaft im Stadt-Umland-Bereich führen. Merkmale dieser Entwicklung sind Arbeitsplatzverluste, steigende Arbeitslosigkeit und sektorale Restrukturierung mit neuen, flexibleren Formen der Produktion und Beschäftigung. Weltweit beobachtete räumliche Konzentrations- bzw. Dekonzentrationstendenzen bestimmter Branchen sind im Wirtschaftsraum Basel nur in geringerem Umfang auszumachen. Regionsspezifische Besonderheiten und die Stellung Basels im nationalen Städtesystem wirken hier modifizierend. Von den Auswirkungen der Restrukturierung sind Stadt und Umland in unterschiedlichem Ausmass betroffen. Auch im Umland selbst vollzieht sich keine homogene Entwicklung. Entsprechend gilt es, die jeweiligen lokalen Stärken auszubauen und Ausgleichsmechanismen zur Sicherung der Wettbewerbsfähigkeit der Region als Ganzes zu implementieren.

\section{Summary: Restructuring of a metropolitan area economy - the case of Basle}

Globalization trends lead to economic processes of restructuring in the City of Basle and its surrounding area. Characteristics of this development are: loss of jobs, increasing unemployment, sectoral restructuring and flexible forms of production and employment. Tendencies towards a spatial concentration or deconcentration of certain economic branches that can be observed on a global scale, can only be found to a smaller degree in the economic region of Basle. Distinctive regional features and Basle's position in the national city-system modify this development. The restructuring processes have different effects on the city and the suburban zone. Within the surrounding areas there is no homogenous development. Therefore, it is necessary to maximize the existing opportunities and to implement equalizing mechanisms that secure the competitiveness of the region as a whole.

\section{Résumé: Processus de restructuration d'une économie suburbaine - Modification du paysage économique régional de Bâle}

Le présent rapport montre que la région de Bâle est également touchée par les tendances de globalisation aboutissant aux processus de restructuration de l'économie dans le domaine suburbain. Les principales caractéristiques de ce développement sont les pertes d'emploi, le chômage croissant, la restructuration sectorielle et de nouvelles formes de production et d'emploi plus souples. Les tendances de concentration et de déconcentration spatiales observées à l'échelle mondiale dans certaines branches n'apparaissent par contre que dans une faible mesure dans l'espace économique de Bâle. Les particularités spécifiquement régionales et la position de Bâle dans le système urbain national ont ici un effet modificateur. Les effets de la restructuration n'ont pas la même portée en ville et en banlieue. Une évolution homogène n'est pas non plus constatée en banlieue même. Il s'agit donc d'exploiter les atouts correspondants et d'implanter des mécanismes compensateurs pour assurer la compétitivité de la région en tant qu'ensemble économique.

Dr. phil. Andrea Kampschulte, Dipl.-Geogr. Renato Strassmann, Abteilung Humangeographie/Stadt- und Regionalforschung, Departement Geographie, Universität Basel, Klingelbergstrasse 16, CH-4056 Basel. 